\title{
Recherche numérique des contacts dans le contexte de la COVID-19
}

\author{
Robert A. Kleinman MD, Colin Merkel MASc
}

- Citation : CMAJ 2020 June 15;192:E653-6. doi: 10.1503/cmaj.200922-f; diffusion hâtive le 27 mai 2020

Voir la version anglaise de l'article ici : www.cmaj.ca/lookup/doi/10.1503/cmaj.200922

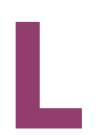

a rapidité avec laquelle le coronavirus du syndrome respiratoire aigu sévère 2 (SRAS-CoV-2) s'est propagé a vite excédé la capacité de nombreuses agences de santé publique d'entreprendre une recherche manuelle des contacts. Pour pallier les limites de la recherche manuelle des contacts, plusieurs gouvernements, dont ceux de l'Alberta, au Canada, de l'Australie, de la France, de l'Allemagne et du Royaume-Uni, ont opté pour la recherche numérique des contacts ${ }^{1-5}$ ou exprimé un intérêt à son endroit. Malgré les avantages de ce type de recherche, avant d'en envisager l'adoption, les agences de santé publique et les gouvernements devraient aussi tenir compte de certains enjeux techniques et des inévitables compromis entre la protection des renseignements personnels et l'efficacité. Sans compter que le succès de la recherche numérique des contacts dépendra de l'accessibilité des tests diagnostiques, de son application à grande échelle et de la capacité des technologies à recenser les expositions. Nous avons analysé les avantages et les inconvénients de la recherche numérique des contacts afin d'informer les gouvernements et les autres parties prenantes et de nourrir leur réflexion sur le bien-fondé de ces nouveaux outils et la façon de les déployer.

\section{Qu'est-ce que la recherche numérique des contacts?}

En temps normal, pour la recherche des contacts, des représentants des autorités sanitaires interrogent une personne infectée, identifient ses contacts et conseillent à ces derniers de surveiller leurs symptômes, de s'astreindre à une quarantaine ou de se soumettre à un examen médical et à un traitement au besoin. Cette approche manuelle a été efficace pour réduire la transmission de nombreuses épidémies, y compris celles de syndrome respiratoire aigu sévère (SRAS) et de maladie à virus Ebola ${ }^{6,7}$. Mais ses limites sont vite devenues apparentes dans le cadre de la pandémie de maladie à coronavirus 2019 (COVID-19). La recherche manuelle des contacts est très exigeante en temps et en ressources humaines, ce qui la rend difficile à appliquer au nombre croissant de personnes infectées par le SRAS-CoV-2. Elle est en outre moins efficace étant donné la brièveté de l'intervalle sériel de la COVID-198. L'imprécision des souvenirs et les expositions survenues dans des endroits publics viennent encore nuire à l'identification des contacts.

\section{POINTS CLÉS}

- La recherche des contacts au moyen d'applications peut combler les limites de la recherche manuelle des contacts sur divers plans : extensibilité, retard des avis, imprécision des souvenirs et identification des contacts dans les endroits publics.

- L'efficacité des applications de recherche des contacts pour l'identification des expositions dépend de leur utilisation à grande échelle et de la capacité des technologies sous-jacentes à identifier les appareils à proximité.

- L'utilisation d'applications de recherche des contacts nécessite certains compromis entre la protection des renseignements personnels et l'efficacité.

- Avant d'être autorisées, les applications doivent être testées sur le terrain dans des conditions réelles pour que l'on puisse en établir la sensibilité et la spécificité dans l'identification des expositions.

- En combinant la recherche manuelle des contacts et la recherche numérique, il est possible de mettre à profit les avantages et d'atténuer les limites de chaque approche.

La recherche numérique des contacts fait appel aux technologies de l'information pour identifier les expositions à l'infection; elle peut pallier certaines lacunes de la recherche manuelle : extensibilité, retard des avis, imprécision des souvenirs et identification des contacts dans les endroits publics.

Plusieurs paradigmes de recherche numérique des contacts sont envisageables. Certains pays, comme la Corée du Sud et la Chine, ont imposé des systèmes de collecte de données à leurs populations, entre autres par l'entremise des images de caméras de surveillance, des transactions financières et des données de localisation des téléphones cellulaires ${ }^{9,10}$. Or, l'imposition de ces systèmes aux participants sans leur consentement et l'atteinte à la vie privée qui en découle les rendent moins acceptables en Amérique du Nord et en Europe. En ce qui concerne les systèmes de recherche numérique sur une base volontaire, ils sont axés sur la création d'applications mobiles à téléchargement volontaire.

La plupart des applications de recherche des contacts dans le contexte de la COVID-19 utilisent l'intensité du signal Bluetooth pour estimer la distance entre les téléphones intelligents et définir un statut d'exposition en fonction de la distance et de la durée de la proximité à une personne qui se révèle infectée par la 
suite. Des applications utilisant la technologie Bluetooth ont été autorisées en Alberta, en Australie et à Singapour à partir d'un cadre conçu par l'Agence gouvernementale des technologies de Singapour ${ }^{11-13}$. D'autres protocoles sont en cours d'élaboration en France et en Suisse ${ }^{14,15}$. En mai 2020, une collaboration entre Apple et Google prévoit proposer une interface de programmation afin d'aider les agences de santé publique à concevoir des applications sur mesure ${ }^{16}$.

Les approches de recherche géoréférencée fonctionnent sans Bluetooth. Elles utilisent plutôt les données des réseaux de téléphonie cellulaire, le système GPS (Global Positioning System), les signaux Wi-Fi et les autres capteurs de signaux des téléphones intelligents pour géolocaliser les utilisateurs des applications, et ces données de localisation permettent de déterminer la proximité des personnes infectées. Les codes à barres représentent une autre stratégie de recherche numérique des contacts. On peut placer des codes QR (Quick Response, codes à barres lisibles par les téléphones) dans des espaces publics, par exemple, sur les portes des autobus et des commerces, et ainsi permettre aux usagers de répertorier les lieux qu'ils ont visités. La recherche à partir des codes QR est utilisée en Chine ${ }^{17}$, mais dans ce pays, si on connaît si bien les codes QR, c'est qu'on les utilise pour les transactions mobiles, et on ignore si cette stratégie peut être implantée en Europe et en Amérique du Nord. Une stratégie connexe repose sur l'empreinte numérique par connexion Wi-Fi, en fonction de l'intensité des signaux reçus à partir de chaque réseau Wi-Fi pour créer une « empreinte » de chaque lieu ${ }^{18}$.

\section{Quelles sont les limites de la recherche des contacts à partir d'applications?}

Les applications de recherche des contacts comportent 5 grandes lacunes. Premièrement, l'efficacité de ces applications dépend de leur adoption par la population : le potentiel d'une telle application correspond au carré de la fraction de ses utilisateurs dans une population ${ }^{19}$. Au cours du premier mois suivant son lancement, l'application de Singapour a été adoptée par $20 \%$ de la population $^{20}$. Une application capable d'identifier $100 \%$ des expositions aurait donc détecté seulement $4 \%$ des contacts. De même, les applications ne peuvent identifier les contacts que si les téléphones de la personne infectée et du sujet exposé sont proches l'un de l'autre et que les 2 utilisent des applications communiquant entre elles. Et quelle que soit l'application de recherche des contacts, elle sera moins efficace dans les communautés où les gens sont moins nombreux à posséder un téléphone intelligent, le partagent, ou sont incapables d'utiliser une application ou réticents à le faire. Le partenariat Apple-Google prévoit lancer " au cours des prochains mois » des mises à jour de leurs systèmes d'exploitation qui permettront l'identification des contacts sur une base volontaire sans télécharger d'application, ce qui accroîtrait l'adoption de la recherche numérique dans les régions qui opteraient pour ce cadre ${ }^{16}$.

Deuxièmement, les technologies qui sous-tendent les applications comportent des erreurs de mesure qui limitent leur efficacité à identifier les contacts. Dans le cas des applications qui font appel à la technologie Bluetooth, il y a plusieurs obstacles associés à l'utilisation de l'intensité du signal pour déterminer la distance entre 2 appareils : l'intensité du signal Bluetooth dépend des appareils, fluctue considérablement et diminue quand les gens se trouvent entre les dispositifs de transmission et de réception ${ }^{13,21,22}$. Un traitement du signal et des tests sur les appareils pourraient atténuer certains de ces obstacles. Les murs et les planchers atténuent aussi les signaux Bluetooth, ce qui a l'avantage potentiel de réduire les fausses expositions. La précision des mesures de géolocalisation est également limitée; lors de tests réalisés à l'extérieur sur un campus universitaire américain, la géolocalisation avec un iPhone 6 connecté à un réseau Wi-Fi a produit des erreurs quadratiques moyennes de 3 à 16 mètres selon le lieu et le moment ${ }^{23}$. Les stratégies reposant sur les codes à barres ont aussi une spécificité spatiale limitée.

Étant donné ces erreurs de mesure, les technologies de recherche numérique des contacts auront une sensibilité et une spécificité inférieures à $100 \%$ pour l'identification des expositions chez les utilisateurs d'applications. Or, autant les expositions passées inaperçues que les fausses expositions ont des conséquences, telles que des expositions évitables ou des quarantaines injustifiées, avec les répercussions psychologiques, financières et sociales qui s'ensuivent. Il faut particulièrement éviter d'identifier à tort des expositions chez les travailleurs de la santé et les premiers répondants qui pourraient se trouver à proximité d'individus infectés tout en portant un équipement de protection individuelle, car leur mise en quarantaine nuirait au fonctionnement du système de santé et à la prestation des soins. Par contre, exclure d'emblée les travailleurs de la santé réduirait l'incidence d'une telle application, étant donné qu'ils peuvent représenter une importante proportion des cas et qu'ils soignent les personnes les plus vulnérables (à qui ils pourraient transmettre l'infection) $)^{24,25}$.

Troisièmement, les approches basées sur la technologie Bluetooth qui n'utilisent pas le système Apple-Google seront limitées par les restrictions de balayage Bluetooth pour les téléphones intelligents fonctionnant avec iOS. Les applications sur iOS peuvent rechercher les appareils Bluetooth à proximité uniquement lorsqu'elles sont visibles à l'écran (à l'avant-plan) ou, quand elles ne sont pas visibles à l'écran (à l'arrière-plan), si le dispositif ciblé est explicitement spécifié26. Étant donné que les dispositifs cibles sont généralement inconnus du système de balayage avant leur détection, cela empêche les appareils qui fonctionnent avec iOS et qui ont des applications de recherche des contacts en arrière-plan de se détecter entre eux. Apple a refusé les demandes de la France et de l'Allemagne de retirer ces restrictions ${ }^{4,27}$. L'Australie aurait trouvé une solution, mais a refusé de la divulguer; la solution recommandée aux utilisateurs à Singapour et en Alberta est de maintenir l'application de traçage à l'avant-plan sur les systèmes iOS ${ }^{1,12}$. Cela vide la batterie plus rapidement et réduit également la sensibilité de l'application si les utilisateurs ne respectent pas cette demande, utilisent d'autres applis ou verrouillent leur écran. Même avec l'approche Apple-Google, les limites de la fréquence de balayage prévues pour limiter la consommation d'énergie nuiront à la détection des expositions brèves. 
Quatrièmement, de manière générale, par rapport à la recherche manuelle de contacts, la surveillance constante et à grande échelle au moyen d'applications de recherche des contacts soulève plus de problèmes en ce qui concerne la protection des renseignements personnels. Les protocoles fondés sur le cadre de Singapour (dont celui de l'Alberta) demandent que tous les utilisateurs s'inscrivent à un serveur central, et que chaque téléphone diffuse des identifiants temporaires et emmagasine les identifiants diffusés par les téléphones à proximité1,2,28. Lorsqu'un utilisateur se révèle positif, la santé publique lui permet de téléverser les identifiants temporaires sauvegardés sur le serveur central, qui les apparie avec les appareils inscrits et fournit l'information à des chercheurs de contacts humains, ce qui ne permet pas l'automatisation des avis aux contacts. Cette approche a été critiquée parce qu'en théorie, elle permet aux gouvernements de reconstruire un " graphique social » des contacts à l'intérieur d'une société29, mais elle leur permet également d'intégrer d'autres renseignements susceptibles de réduire l'identification de fausses expositions. Le cadre Apple-Google propose une structure de données décentralisée pour protéger les renseignements personnels et empêcher les gouvernements d'établir un réseau de contacts sociaux. Chaque téléphone émet des identifiants variables provenant de clés cryptographiques changeantes et conserve les identifiants émis par les appareils à proximité ${ }^{30}$. Lorsqu'un utilisateur se révèle positif, la santé publique lui offre le choix de téléverser ses clés cryptographiques antérieures sur un serveur central; ces clés sont alors diffusées à tous les appareils, ce qui permet un appariement local (sur les appareils) avec les identifiants reçus. Les agences de santé publique ne reçoivent que les clés transmises volontairement par les personnes infectées, avec le jour et la durée de l'exposition. Même si une telle approche limite la capacité des gouvernements de dépersonnaliser les données et d'établir les réseaux de contacts, elle limite aussi la capacité des agences de santé publique de faire le point sur les recommandations aux fins de contrôle de la qualité, de vérifier l'exactitude des avis d'exposition et d'appliquer des initiatives d'amélioration de la qualité.

La collecte des données de géolocalisation a d'autres conséquences : elle permettrait aux organisations de recueillir des données sur les déplacements des personnes et donc de faire des inférences quant à leurs habitudes et leurs préférences. Même les données de localisation dépersonnalisées ne peuvent être entièrement anonymisées; des recoupements peuvent être faits entre les coordonnées géographiques et d'autres dossiers des services publics et permettre la création de modèles probabilistes concernant les personnes à qui elles appartiennent.

Cinquièmement, même si des études de modélisation ont laissé entendre que les applications de recherche des contacts permettraient de réduire la transmission ${ }^{19}$, aucune étude n'a été publiée en ce sens. Si les applications donnent lieu à un relâchement du respect des autres mesures de prévention, ou à une baisse de la collaboration pour la recherche habituelle des contacts, leur utilité s'en trouverait amoindrie, voire négative.

\section{Quelle est la meilleure voie à suivre pour la recherche numérique des contacts?}

\section{Intégrer la recherche numérique et la recherche manuelle des contacts}

En Corée du Sud, une recherche manuelle des contacts de chaque cas a été réalisée, vérifiée et complétée par le contenu des dossiers médicaux, des images de caméra de surveillance, des données de géolocalisation des téléphones cellulaires et des transactions financières, afin de créer un rapport détaillé des déplacements récents des personnes infectées ${ }^{9}$. Des renseignements détaillés sur les déplacements de chaque personne infectée ont ensuite été envoyés par message texte aux personnes qui pourraient s'être trouvées à proximité d'elle. Il est peu probable que cette stratégie soit acceptée au Canada en raison des risques d'atteinte à la vie privée. Alors, comment adapter cette approche au contexte canadien?

La recherche manuelle des contacts devrait être reconnue comme une façon très spécifique d'identifier les expositions et devrait être substantiellement élargie compte tenu de l'ampleur et de l'urgence de la situation. La technologie peut ensuite être mise à contribution pour améliorer l'efficience de la collecte des données, automatiser une portion des avis aux contacts et soutenir les personnes placées en quarantaine ${ }^{31}$.

En plus de monter des registres de contacts, les applications peuvent permettre aux utilisateurs de sauvegarder de manière optionnelle leurs données de localisation sur leur téléphone, ce qui peut leur servir d'aide-mémoire lors de la recherche manuelle des contacts ${ }^{32}$. Pour réduire encore plus les erreurs de rappel, les personnes infectées peuvent être encouragées à passer en revue volontairement leurs dossiers numériques, par exemple, leurs transactions financières. Ensemble, ces 2 stratégies feraient des données numériques un complément à la recherche manuelle des contacts, tout en assurant la protection de la vie privée et le caractère optionnel des mesures.

Pour réduire les décalages entre l'identification des cas et les avis aux contacts, des applications peuvent être déployées comme outils de dépistage rapide, en misant sur des seuils de distance et de durée pour définir un ensemble de critères d'exposition et permettre une détection sensible des contacts. Les avis pourraient être envoyés rapidement et automatiquement aux contacts potentiels, en recommandant l'isolement jusqu'à la réalisation d'un suivi plus spécifique par recherche manuelle des contacts. Une telle approche atténuerait l'imprécision de ces technologies, mais au prix d'un certain nombre de fausses expositions à court terme. Avec l'augmentation du nombre de cas, l'adoption progressive de seuils plus sensibles pourrait aider à contenir le SRAS-CoV-2.

\section{Protection des renseignements personnels}

En plus d'utiliser une stratégie de recherche des contacts qui assure la protection des renseignements personnels, les applis devraient être dotées de caractéristiques protégeant la vie privée conformes aux lois fédérales et provinciales et aux pratiques internationales optimales énoncées dans le Règlement général sur la protection des données de l'Union européenne. 
Ces caractéristiques sont notamment le cryptage de toutes les données personnelles, les explications en langage simple et le consentement des utilisateurs à la conservation et à l'utilisation des données, les restrictions quant à l'utilisation des données en dehors des mesures de la santé publique contre la COVID-19, l'élimination automatique des données et l'option d'élimination des données en tout temps. (L'élimination des données protège les renseignements personnels et peut apaiser les craintes concernant la surveillance susceptibles de nuire à l'adoption des technologies; toutefois, elle limite la capacité de vérifier a posteriori l'efficacité de l'application et les possibilités de l'améliorer.) L'utilisation d'une application devrait être volontaire, et les utilisateurs devraient avoir l'option de mettre la recherche des contacts sur pause, tant pour protéger leurs renseignements personnels que pour permettre aux travailleurs de la santé de désactiver la surveillance lorsqu'ils utilisent des précautions appropriées.

\section{Mise à l'essai sur le terrain et publication des résultats}

Avant d'être autorisées, les applications doivent être mises à l'essai sur le terrain dans des conditions concrètes pour comprendre les caractéristiques de fonctionnement du récepteur. Les seuils de distance et de durée définissant une exposition doivent être sélectionnés en fonction de critères combinés de sensibilité et de spécificité. La sensibilité et la spécificité de chaque application et du cadre Apple-Google devraient être publiées pour permettre aux agences de santé publique de décider en toute connaissance de cause, le cas échéant, quoi déployer.

\section{Promouvoir l'adoption}

Étant donné que l'efficacité des applications de recherche des contacts dépend largement de leur adoption à grande échelle, les gouvernements devraient en encourager l'utilisation si les tests sur le terrain indiquent qu'elles permettent d'identifier les expositions de manière fiable. Les applications peuvent également être efficaces dans certaines sous-populations qui comptent une forte proportion d'utilisateurs. II faudrait particulièrement en encourager l'adoption dans les sous-groupes exposés à des situations pour lesquelles la recherche manuelle des contacts ne convient pas, par exemple, les usagers des transports en commun, les clients des épiceries et, lors de la levée des restrictions, les participants à des rencontres professionnelles, sociales et publiques. L'adoption devrait aussi être encouragée chez les travailleurs hospitaliers, le personnel des établissements de soins de longue durée et des établissements correctionnels et les autres personnes qui travaillent auprès de gens à risque d'être gravement atteints de la COVID-19. Pour assurer un accès équitable et améliorer l'efficacité de la recherche des contacts, les gouvernements devraient fournir des dispositifs à faible coût aux personnes qui n'ont pas de téléphones intelligents avec connexion Bluetooth. Dans les régions servies par différentes agences de santé publique et là où il y a beaucoup de déplacements entre les régions, par exemple, entre les provinces canadiennes ou entre les États des États-Unis, la coordination entre les agences de santé publique sera nécessaire pour identifier les expositions entre les utilisateurs d'applications différentes.

\section{Accroître l'accès aux tests diagnostiques}

Étant donné que la recherche numérique des contacts dépend de l'identification des personnes porteuses du SRAS-CoV-2, il sera crucial d'élargir l'accès à des tests diagnostiques de haute sensibilité pour en assurer la réussite. La réalisation de tests chez tous les individus potentiellement exposés selon une application pourrait aider à identifier les cas asymptomatiques. Compte tenu du court intervalle sériel de la COVID-19, il sera important d'accélérer l'obtention des résultats et d'abréger l'intervalle entre le déclenchement des symptômes, l'identification des contacts et la réalisation des tests pour assurer la réussite de la recherche des contacts.

\section{Conclusion}

Avant de se prononcer sur le bien-fondé des technologies de recherche numérique des contacts, les agences de santé publique et les gouvernements doivent être bien au fait de leurs avantages potentiels, des enjeux techniques et des compromis inévitables entre la protection des renseignements personnels et l'efficacité. Les seuils de sensibilité et de spécificité des outils numériques, établis après une mise à l'essai en situation réelle et avant leur autorisation, doivent être établis en fonction de l'usage auquel ils sont destinés. La réussite de la recherche numérique des contacts dépendra de l'accès aux tests diagnostiques, de son adoption à grande échelle et de la capacité des technologies à identifier les expositions. En combinant la recherche manuelle des contacts et la recherche numérique, il est possible de mettre à profit les avantages et d'atténuer les limites de chaque approche.

\section{Références}

1. ABTraceTogether FAQ. Government of Alberta; 2020. Accessible ici : www. alberta.ca/ab-trace-together-faq.aspx (consulté le 2 mai 2020).

2. COVIDSafe app. Canberra (AU): Australia Australian Government Department of Health; 2020. Accessible ici : www.health.gov.au/resources/apps-and-tools/ covidsafe-app (consulté le 30 avril 2020).

3. Sportisse B. "Contact tracing": Bruno Sportisse, CEO of Inria, gives some elements to better understand the challenges. [article en français]; mis à jour le 23 avril 2020. Accessible ici : www.inria.fr/sites/default/files/2020-04/ Contact $\% 20$ tracing\%2C\%20quelques\%20\%C3\%A91\%C3\%A9ments\%20pour $\% 20$ mieux\%20comprendre\%20les\%20enjeux.pdf (consulté le 25 avril 2020).

4. Busvine D, Rinke A. Germany at odds with Apple on smartphone coronavirus contact tracing. Reuters 23 avril 2020. Accessible ici : www.reuters.com/article/ us-health-coronavirus-europe-tech-idUSKCN2251MR (consulté le 25 avril 2020).

5. Gould M, Lewis G. Digital contact tracing: protecting the NHS and saving lives. London (UK): NHSX [National Health Service]; 24 avril 2020. Accessible ici : www.nhsx.nhs.uk/blogs/digital-contact-tracing-protecting-nhs-and-savinglives/ (consulté le 25 avril 2020).

6. Svoboda T, Henry B, Shulman L, et al. Public health measures to control the spread of the severe acute respiratory syndrome during the outbreak in Toronto. N Engl J Med 2004;350:2352-61.

7. Swanson KC, Altare C, Wesseh CS, et al. Contact tracing performance during the Ebola epidemic in Liberia, 2014-2015. PLoS Negl Trop Dis 2018;12:e0006762.

8. Zhang J, Litvinova M, Wang W, et al. Evolving epidemiology and transmission dynamics of coronavirus disease 2019 outside Hubei province, China: a descriptive and modelling study. Lancet Infect Disease 2 avril 2020 [Cyberpublication avant impression]. S1473-3099(20)30230-9. doi: 10.1016/S1473-3099(20)30230-9.

9. COVID-19 National Emergency Response Center, Epidemiology \& Case Management Team, Korea Centers for Disease Control \& Prevention. Contact transmission of COVID-19 in South Korea: novel investigation techniques for tracing contacts. Osong Public Health Res Perspect 2020;11:60-3.

10. Yang Y, Zhu J. Coronavirus brings China's surveillance state out of the shadows. Reuters 7 février 2020. Accessible ici : www.reuters.com/article/us-china -health-surveillance-idUSKBN2011HO (consulté le 25 avril 2020). 
11. Toy, A. Alberta's contact-tracing app only works on iOS when phone is unlocked, app running in foreground. Global News 4 mai 2020, mis à jour le 5 mai 2020. Accessible ici : https://globalnews.ca/news/6898691/ab-tracetogether-contact-app-alberta-covid-ios/ (consulté le 21 mai 2020).

12. Bogle A. COVIDSafe's effectiveness on iPhone in question as Government releases coronavirus contact tracing app. ABC News 26 avril 2020. Accessible ici : www.abc.net.au/news/2020-04-26/coronavirus-tracing-app-covidsafe -apple-iphone-covid-19/12187448 (consulté le 2 mai 2020).

13 TraceTogether - behind the scenes look at its development process. Singapore: Government Technology Agency; 2020. Accessible ici : www.tech.gov.sg/ media/technews/tracetogether-behind-the-scenes-look-at-its-development -process (consulté le 5 avril 2020).

14. ROBERT: ROBust and privacy-presERving proximity Tracing. France: PRIVATICS team (Inria) and Germany: Fraunhofer AISEC; 2020. Accessible ici : https:// github.com/ROBERT-proximity-tracing/documents/blob/master/ROBERT -specification-EN-v1_0.pdf (consulté le 25 avril 2020).

15. EPFL and ETH Zurich advance digital contact tracing project [news article]. Lausanne, (Switzerland): École polytechnique fédérale de Lausanne (EPFL); 2020. Accessible ici : https://actu.epfl.ch/news/epfl-and-eth-zurich-advance-digital -contact-tracin/ (consulté le 25 avril 2020).

16. Apple and Google partner on COVID-19 contact tracing technology. Apple Newsroom 2020. Accessible ici : www.apple.com/newsroom/2020/04/apple -and-google-partner-on-covid-19-contact-tracing-technology/ (consulté le 25 avril 2020).

17. Shanghai offers health QR codes in public transport. Xinhua [Beijing, China] 2020. Accessible ici : www.xinhuanet.com/english/2020-02/25/c_138817911. htm (consulté le 6 avril 2020).

18. He S, Chan S-HG. Wi-Fi fingerprint-based indoor positioning: recent advances and comparisons. IEEE Comm Surv and Tutor 2016;18:466-90.

19. Ferretti L, Wymant C, Kendall M, et al. Quantifying SARS-CoV-2 transmission suggests epidemic control with digital contact tracing. Science 2020;eabb6936.

20. Team TraceTogether. 20 April 2020 - one month on. Singapore: Government Technology Agency; 2020. Accessible ici : http://tracetogether.zendesk. com/hc/en-sg/articles/360046475654 (consulté le 3 mai 2020).

21. Li G, Geng E, Ye Z, et al. Indoor positioning algorithm based on the improved RSSI distance model. Sensors (Basel) 2018;18:2820.

22. Cotton SL. Human body shadowing in cellular device-to-device communications: channel modeling using the shadowed \$|kappa-।mu\$ fading model. IEEE J Sel Areas Comm 2015;33:111-9.
23. Merry K, Bettinger P. Smartphone GPS accuracy study in an urban environment. PLoS One 2019;14:e0219890.

24. CDC COVID-19 Response Team. Characteristics of health care personnel with COVID-19 - United States, February 12-April 9, 2020. MMWR Morb Mortal Wkly Rep 2020;69:477-81.

25. COVID-19 in Canada: modelling update. Ottawa: Public Health Agency of Canada; 2020. Accessible ici : www.canada.ca/content/dam/phac-aspc/documents/ services/diseases/2019-novel-coronavirus-infection/using-data-modelling -inform-eng-04-28.pdf (consulté le 3 mai 2020).

26. scanForPeripheralsWithServices:options: In: Apple developer guide. Cupertino (CA): Apple; 2020. Accessible ici : https://developer.apple.com/documentation/ corebluetooth/cbcentralmanager/1518986-scanforperipheralswithservices (consulté le 6 avril 2020).

27. Fouquet $\mathrm{H}$. France says Apple bluetooth policy is blocking virus tracker. Bloomberg 20 avril 2020, mis à jour le 21 avril 2020. Accessible ici : www. bloomberg.com/news/articles/2020-04-20/france-says-apple-s-bluetooth -policy-is-blocking-virus-tracker (consulté le 25 avril 2020).

28. Bay J, Kek J, Tan A, et al. BlueTrace: A privacy-preserving protocol for communitydriven contact tracing across borders. BlueTrace Protocol; 2020. Accessible ici : https://bluetrace.io/static/bluetrace_whitepaper-938063656596c104632def383 eb33b3c.pdf (consulté le 21 mai 2020).

29. Multiple signatories. Joint statement on contact tracing. 19 avril 2020. Accessible ici : www.esat.kuleuven.be/cosic/sites/contact-tracing-joint-statement/ (consulté le 25 avril 2020).

30. Exposure notification: cryptography specification: preliminary - subject to modification and extension. Apple/Google; 2020. Accessible ici : https:// covid19-static.cdn-apple.com/applications/covid19/current/static/contact -tracing/pdf/ExposureNotification-CryptographySpecificationv1.1.pdf (consulté le 25 avril 2020).

31. Jung A. Here's how technology is helping researchers and health officials connect with quarantined patients. CTV News Vancouver 6 mars 2020. Accessible ici : https://bc.ctvnews.ca/here-s-how-technology-is-helping-researchers-and -health-officials-connect-with-quarantined-patients-1.4843038 (consulté le 13 mai 2020).

32. Hart V, Siddarth D, Cantrell B, et al. Outpacing the virus: digital response to containing the spread of COVID-19 while mitigating privacy risks. Cambridge (MA): Harvard University, Edmond J. Safra Center for Ethics; 3 avril 2020 Accessible ici : https://ethics.harvard.edu/files/center-for-ethics/files/white_ paper_5_outpacing_the_virus_final.pdf (consulté le 5 avril 2020).
Intérêts concurrents : En mars et au début d'avril, les auteurs ont mis au point un prototype d'application de recherche des contacts par géolocalisation. Ils déclarent ne pas avoir et n'avoir jamais eu d'intérêt commercial pour le développement de ladite application (et ne travaillent plus à ce projet). Les auteurs transmettront gratuitement leur travail à toute agence de santé publique qui le demande. Colin Merkel dit être un ingénieur en logiciels qui a travaillé chez Google jusqu'à juin 2019. La filiale de Google, Alphabet, fabrique les systèmes d'exploitation Android, et Google produit des services de cartographie. Colin Merkel travaille maintenant pour une compagnie en démarrage dans le domaine de l'éducation. Il n'a reçu aucune rémunération pour ce travail. Aucun autre intérêt concurrent n'a été déclaré.
Cet article a été révisé par des pairs.

Affiliations : Faculté de médecine de l'Université Stanford (Kleinman), Stanford, Californie; ingénieur en logiciels (Merkel), Toronto, Ont.

Collaborateurs : Les 2 auteurs ont contribué à l'élaboration et à la conception des travaux. Robert Kleinman a rédigé la première version du manuscrit. Les 2 auteurs ont révisé de façon critique le contenu intellectuel important du manuscrit; ils ont donné leur approbation définitive pour la version destinée à être publiée et assument l'entière responsabilité de tous les aspects du travail.

Correspondance : Robert Kleinman, rkleinman@stanford.edu 